\title{
Can Improved Periodontal Health Be a Key Factor in Preventing Severe COVID-19 Complications: An Evidence-Based Review
}

\section{Vindeshwari Bhatia*, Ashadeep, Ajay Mahajan, Kanwarjit Singh Asi \\ Department of Periodontology, Himachal Pradesh Government Dental College and Hospital, Shimla, Himachal Pradesh, India-171001}

*Correspondence to: Vindeshwari Bhatia, Department of Periodontology, Himachal Pradesh Government Dental College and Hospital, Shimla, Himachal Pradesh, India-171001; E-mail: vindubhatiaggt@gmail.com

Received date: February 7, 2021; Accepted date: February 18, 2021; Published date: February 25, 2021

Citation: Bhatia V, Ashadeep, Mahajan A, et al. (2021) Can Improved Periodontal Health Be A Key Factor In Preventing Severe COVID-19 Complications: An EvidenceBased Review. Advanc Dentistry 2(1): pp. 1-4.

Copyright: @2021 Bhatia V. This is an open-access article distributed under the terms of the Creative Commons Attribution License, which permits unrestricted use, distribution, and reproduction in any medium, provided the original author and source are credited.

\section{ABSTRACT}

COVID-19 is now recognized as a pandemic throughout the world and the severity of the disease has been attributed to many patient-related medical risk factors, which include diabetes, hypertension, respiratory diseases, cardiovascular disease and chronic kidney diseases due to exaggerated immune response associated with these diseases. However, in recent decades, there has been a renewed interest in the association of systemic diseases with periodontal health as the severity of these diseases increases in patients having compromised periodontal health. Therefore, it is suggested that there could be a possible link between the status of periodontal health and the severity of the disease in patients suffering from COVID-19. The purpose of this review article is to explore and highlight the various common mechanisms between periodontal disease and COVID-19 and further explore the role of periodontal disease as a contributing factor for COVID-19 related complications.

\section{Keywords:}

COVID-19, Comorbidities, Oral health, Periodontal diseases

\section{Introduction}

The COVID 19 is an ongoing global pandemic, caused by Severe Acute Respiratory Syndrome Coronavirus-2 (SARS CoV2) [1]. The outbreak was first identified in December 2019 in Wuhan, China. World Health Organization has declared the outbreak a Public Health Emergency of International Concern on 30 January 2020 and as a pandemic on 11 March [2]. As of 5 January 2021, more than 86.4 million cases of COVID 19 have been reported worldwide resulting in more than 1.87 million deaths [3]. Although the global death-to-case ratio is 3.7 percent of the total confirmed cases [4], the risk of severe illness and death has been highest in older people and in certain cases with underlying non-communicable diseases (NCDs), such as chronic lung disease, hypertension, cardiac disease, diabetes and cancer [5-8]. It should come as no surprise that the majority of these diseases have been directly or indirectly linked to periodontal health as it is well-established that, the severity of these conditions increases in the patients having compromised periodontal health, which could be associated with exaggerated host immune response $[9,10]$. Therefore, it would be interesting to theorize that there could be a possible link between the status of periodontal health and the severity of the disease in patients suffering from COVID-19. The present article explores and highlights the various mechanisms to investigate the role of periodontal disease as a contributing factor for COVID-19 related complications.

\section{Search strategy}

A literature search for published articles was carried out using electronic and manual searches. A search of electronic databases, including PUBMED, MEDLINE up to 2020 was made.
Key terms used in the search included risks of and pathogenesis of COVID-19, COVID-19 and comorbidities, Periodontal disease and systemic conditions, Oral health and COVID-19, Periodontal disease a risk factor for severe COVID-19 illness.

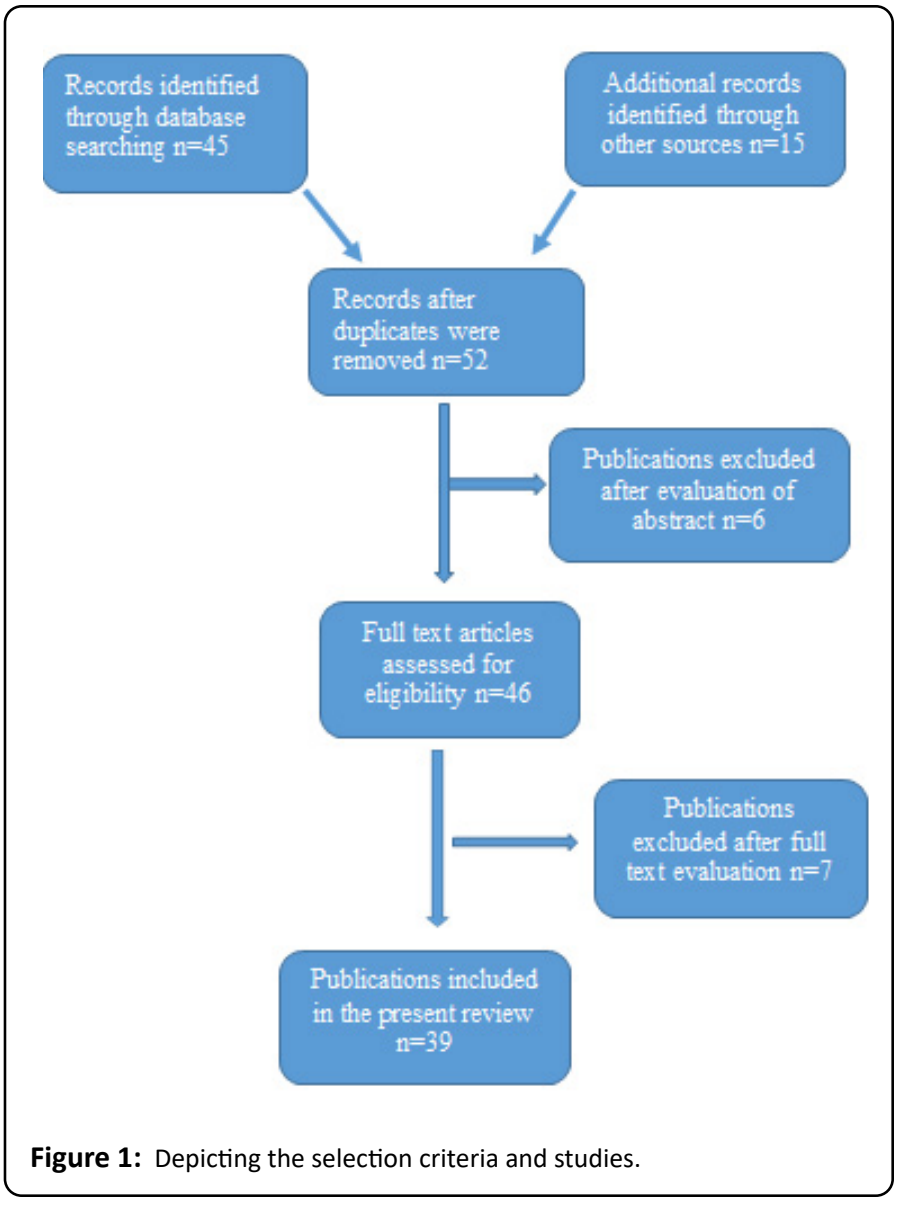


Citation: Bhatia V, Ashadeep, Mahajan A, et al. (2021) Can Improved Periodontal Health Be A Key Factor In Preventing Severe COVID-19 Complications: An Evidence-Based Review. Advanc Dentistry 2(1): pp. 1-4.

\section{Periodontal disease and systemic disease}

Periodontal disease is an inflammatory disease in which microbial etiologic factors induce a series of host responses that mediate inflammatory events, leading to tissue destruction in susceptible individuals [11]. The recent Global Burden of Disease Study (GBD,1990-2010) indicates that periodontitis is the 6th most prevalent disease worldwide, with an overall prevalence of $11.2 \%$ [12]. Although bacterial plaque is the main causative agent responsible for inducing the host inflammatory process but it is the host susceptibility and ability of the host defense to respond appropriately to the bacterial confront in periodontal diseases [13-15]. Furthermore, the relationship between periodontal disease and systemic health is well documented as a bilateral relationship, in which the local bacterial challenge producing an extensive amount of inflammatory mediators with the potential to induce adverse systemic outcomes and the systemic host factors acting locally to reduce resistance to periodontal destruction [9]. Therefore, it is evident that several factors, including systemic diseases and conditions, influence individual susceptibility to periodontitis.

Periodontal lesions are recognized as a reservoir of gramnegative bacteria, inflammatory mediators such as proinflammatory cytokines and microbial toxins that could enter the bloodstream and be a risk factor for diabetes mellitus, cardiovascular disease, respiratory disease, rheumatoid arthritis, and others [9-11].

DM is a metabolic disorder characterized by hyperglycemia due to defective secretion or activity of insulin. Periodontal pathogens can invade deep vascular endothelium about the periodontium and further aggravate the microvascular complications associated with the DM by releasing their toxins and metabolic products [16].

The presence of periodontal pathogens in atheromatous plaque and their association with atherogenesis have been shown by various studies. These microbes lead to atherogenesis either by direct invasion of the arterial wall or by the release of inflammatory mediators with atherogenic effects. Moreover, increased levels of CRP, TNF- $\alpha$ also provide insight into the association of PD with hypertension and CVD [17].

COPD is a combination of chronic bronchitis and emphysema and literature has shown that periodontitis increases the risk of developing COPD. Accumulation and activation of neutrophils is the common pathophysiological mechanism in both the diseases. Factors released by neutrophil granules cause damage to the connective tissue. Moreover, elevated levels of C Reactive Proteins (CRP), Interleukin-8 (IL-8), Tumor Necrosis Factor- $\alpha$ (TNF- $\alpha$ ), are also confirmed in both diseases [18].

\section{COVID -19 and its impact on systemic diseases}

COVID-19 involves systemic inflammation with several consequences due to the direct effect of the virus on almost all body organs and widespread inflammatory response. Once the pathogen (SARS-CoV-2) enters the human body, it invades the alveoli and links to the Angiotensin-Converting Enzyme 2 (ACE2) receptor expressed by lung alveolar cells through their spike protein and induces hyaline membrane formation in the alveoli, followed by interstitial widening and edema. These structural changes result in the alteration in the alveolar- capillary exchange, which further leads to severe respiratory distress $[5,7]$.

After the respiratory system, the second most affected body system is the cardiovascular system as SARS-CoV-2 exhibits affinity towards ACE 2 receptors, which are widely spread in the cardiovascular system and may have a role in heart injury. Moreover, overproduction of immune cells called a cytokine storm triggered by imbalanced response of helper $T$ cells and respiratory dysfunction with hypoxemia may lead to myocarditis and acute heart failure $[5,19,20]$.

The expression of ACE 2 may be substantially increased in patients with type 1 or type 2 diabetes and in the brush border of proximal tubular cells of the kidney. Therefore, it is suggested that having increased levels of ACE-2 can facilitate the infection with COVID-19 as storms of inflammatory responses and higher levels of cytokines produced in the disease are intimately involved in the more severe degrees of complications in patients with diabetes and Chronic Kidney Disease (CKD) [6,21].

\section{Periodontal disease as a risk factor for covid-19}

Plenty of evidence in the literature suggests that periodontitis is associated with several chronic systemic diseases and these systemic diseases have been associated with severe COVID-19 illness $[5,8,15]$. There is also evidence in the literature suggesting the association between periodontitis and COVID-19 related adverse outcomes $[22,23]$. Therefore, it is possible that periodontitis could be associated with severe COVID-19 complications. Following are the possible mechanisms linking periodontal disease as a contributing factor for COVID-19 related complications:

\section{Gingival sulcus: a potential niche for SARS-CoV-2}

Search for viral loads in various body fluids has been done in the literature of which samples of saliva, urine, breast milk, and peritoneal fluid have demonstrated the presence of SARS-CoV-2 [24]. Gingival Crevicular Fluid (GCF) is an inflammatory exudate generated in periodontal tissues that drains into the gingival sulcus to flush the subgingival space and carries within it molecular and cellular components such as antibodies, plasma cells, neutrophils, and components of the complement system. A recent study has confirmed the presence of SARS-CoV- 2 in the GCF of the patients with periodontal diseases and from this study, it can be inferred that virus could find a favorable environment to survive and replicate in the periodontal pocket and to reach continuously the oral cavity and mix with saliva, or to migrate systemically using periodontal capillary complex [24].

As the gingival sulcus is considered to be a conventional niche for microbes and their association with respiratory diseases, therefore, the symbiotic relationship between the microbes could be associated with high viral proliferation into the gingival sulcus $[25,26]$. Moreover, the presence of ACE2 which is the major receptor for SARS-CoV-2 in the epithelium of salivary glands and gingiva it can be hypothesized that the gingival sulcus is a potential ecological niche for the SARSCoV-2 [25]. Furthermore, the presence of periodontopathic bacteria in the metagenome of patients infected with novel coronavirus has been demonstrated with higher levels of 
Citation: Bhatia V, Ashadeep, Mahajan A, et al. (2021) Can Improved Periodontal Health Be A Key Factor In Preventing Severe COVID-19 Complications: An Evidence-Based Review. Advanc Dentistry 2(1): pp. 1-4.

Provetella, fusobacterium, and staphylococcus [27]. This would lead one to postulate that increased viral load in patients with periodontal diseases may aggravate the severe complications associated with SARS-CoV-2 as the viral load has already been proven to be directly proportional to severe COVID-19 complications $[28,29]$.

\section{COVID-19 and periodontitis: The cytokine connection}

Novel coronavirus was recently named as a "hit and run" virus, which causes an alteration in the immune system, leading to autoimmune damage. Cytokine storm is caused by dysregulated humoral and cellular mechanisms and characterized as elevated serum levels of IL-2, IL-6, IL-10, TNF, and C-reactive protein $[27,30]$. Higher levels of these inflammatory markers are associated with the more severe outcomes of COVID-19 infection. Moreover, elevated Th17 pathway responses have also been observed in COVID-19 infected patients which are involved in the manifestation of the cytokine storm and adverse outcomes pertaining to pulmonary oedema and tissue damage in the lungs [31,32].

Similarly, pro-inflammatory mediators such as cytokines (IL-1, TNF) produced in diseased periodontal tissues can infiltrate the saliva and be aspirated to cause alteration in respiratory epithelium and reduce the airflow [27]. Moreover, there has also been the demonstration of increased IL-17 producing cells in gingival tissues of patients suffering from periodontal diseases [32]. Therefore, this common pathway of inflammatory response points towards a possible association between periodontitis and COVID-19 related complications.

\section{Neutrophils extracellular traps: a new suggested commonality} between covid 19 and periodontal diseases

One of the mechanisms of neutrophil action is the formation of neutrophil extracellular traps (NETs). NETs are composed of nuclear chromatin in decondensed form, that act as scaffolds, ideal for retaining microbes [33]. The main function of NETs is trapping and killing pathogens because of their association with antimicrobial proteins mainly the histones and peptides $[33,34]$. The process of NETs generation, called NETosis, is a multistep cell death pathway different from apoptosis and necrosis. The involvement of NETosis in various diseases has been established, in particular autoimmune diseases, cancer, diabetes [35,36]. Also, a validated role of NETosis in the pathophysiology of periodontal diseases has been found, as levels of interferon-alpha, the main mediator involved in the stimulation of NETs release is reported higher in patients with periodontal diseases [37].

Moreover, viruses are well known to evade the host defense as suggested with emerging evidence that points towards their action as triggers of NETosis [38]. Consequently, the virus-induced NETosis, implied to be strongly associated with exaggerated inflammatory and immunological response owing to the NETs circulation in the body in an uncontrolled way, leading to the extreme systemic response of the host with the increased production of immune complexes, cytokines, and chemokines [39]. Therefore, this new intriguing commonality of increased levels of NETs in both periodontal diseases and COVID-19 would point towards the possibility of severe COVID-19 related complications in patients with periodontal diseases.

\section{Conclusion}

The more severe the form of COVID-19, the higher will be the chances of complications associated with it. The severity of complications in COVID-19 patients depends on various risk factors. There is emerging evidence to support that periodontal diseases could be a potential risk factor for COVID-19 infection because of the association of periodontal diseases with various systemic diseases and conditions. Therefore, a strong connecting link between viral load in periodontal diseases and severity of COVID-19 illness can be suggested but future studies on the periodontal status of patients with COVID-19 are still needed to substantiate these findings. Moreover, understanding this association further underscores the importance of keeping the periodontal disease status under check in the clinical management of COVID-19 patients because improved periodontal health may play a role in reducing the risk of severe COVID-19 complications.

\section{References}

1. Sharma A, Tiwari S, Kanti M, et al. (2020) Since January 2020 Elsevier has created a COVID-19 resource center with free information in English and Mandarin on the novel coronavirus COVID- 19. The COVID-19 resource center is hosted on Elsevier Connect, the company's s public news and information. Science 80.

2. WHO (2020) COVID-19 Public Health Emergency of International Concern (PHEIC) Global research and innovation forum. World Heal Organ 7.

3. https://www.who.int/publications/m/item/weeklyepidemi ological-update---5-january-2021

4. World Health Organization (2020) Coronavirus disease (COVID-19) Situation Report-198. A A Prac 14: pp. e01218

5. Temgoua MN, Francky Teddy Endomba FT, Nkeck JN, et al. (2020) Coronavirus Disease 2019 ( COVID-19) as a MultiSystemic Disease and its Impact in Low- and Middle-Income Countries ( LMICS ). SN Compr Clin Med 2020: pp. 1-11.

6. Schofield J, Leelarathna L, Thabit H (2020) COVID-19: Impact of and on Diabetes. Diabetes Ther 11(7): pp. 1429-1435.

Ejaz H, Alsrhani A, Zafar A, et al. (2020) COVID-19 and comorbidities : Deleterious impact on infected patients. J Infect Public Health 13(12): pp. 1833-1839.

8. Richardson S, Hirsch JS, Narasimhan M, et al. (2020) Presenting Characteristics, Comorbidities, and Outcomes Among 5700 Patients Hospitalized With COVID-19 in the New York City Area. JAMA 323(20): pp. 2052-2059.

9. Arigbede AO, Babatope BO, Bamidele MK (2012) Periodontitis and systemic diseases: A literature review. J Indian Soc Periodontol 16(4): pp. 487-491.

10. Bui FQ, Coutinho Almeida-da-Silva CL, Huynh B, et al. (2019) Association between periodontal pathogens and systemic disease. Biomed J 42(1): pp. 27-35.

11. Cekici A, Kantarci A, Hasturk H, et al. (2014) Inflammatory and immune pathways in the pathogenesis of the periodontal disease. Periodontol 2000 64(1): pp. 57-80.

12. Tonetti MS, Jepsen S, Jin L, et al. (2017) Impact of the global burden of periodontal diseases on health, nutrition and wellbeing of mankind: A call for global action. J Clin Periodontol 
Citation: Bhatia V, Ashadeep, Mahajan A, et al. (2021) Can Improved Periodontal Health Be A Key Factor In Preventing Severe COVID-19 Complications: An Evidence-Based Review. Advanc Dentistry 2(1): pp. 1-4.

44(5): 456-462.

13. Guthmiller JM, Novak KF (2002) Polymicrobial DiseaseChapter 8 Periodontal Diseases. Am Soc Microbiol pp. 1-16.

14. Cekici A, Kantarci A, Hasturk H, et al. (2014) Inflammatory and immune pathways in the pathogenesis of the periodontal disease. Periodontol 2000 64(1): pp. 57-80.

15. Rodríguez E (1989) We are IntechOpen, the world's leading publisher of Open Access books Built by scientists, for scientists TOP 1\%. Intech 32(1): 137-144.

16. Sima C, Glogauer M (2013) Diabetes mellitus and periodontal diseases. Curr Diab Rep 13(1): pp. 445-452.

17. Sanz M, Del Castillo AM, Jepsen S, et al. (2020) Periodontitis and cardiovascular diseases: Consensus report. J Clin Periodontol 47(3): pp. 268-288.

18. Gomes-Filho IS, da Cruz SS, Trindade SC, et al. (2020) Periodontitis and respiratory diseases: A systematic review with meta-analysis. Oral Dis 26(2): pp. 439-446.

19. Zhang J, Wang $X$, Jia $X$, et al. (2020) Risk factors for disease severity, unimprovement, and mortality in COVID-19 patients in Wuhan, China. Clin Microbiol Infect 26(6): pp. 767-772.

20. Minga IG, Golemi L, Pursnani A (2020) The Novel Coronavirus Disease (COVID-19) and Its Impact on Cardiovascular Disease. Cardiol Rev 28(4): pp. 163-176.

21. Dadson P, Tetteh CD, Rebelos E, et al. (2020) Underlying Kidney Diseases and Complications for COVID-19: A Review. Front Med 7(1): pp. 4-9.

22. Pitones-rubio V, Hurtado-camarena A, González-rascón $A$ (2020) Is periodontal disease a risk factor for severe COVID-19 illness ? Med Hypotheses 144(8): pp. 109969.

23. Froum $\mathrm{S}(2020)$ Can periodontal disease be a contributing factor for COVID-19 severity? 1-17 https:// www.perioimplantadvisory.com/clinical-tips/periodontalcomplications/article/14179112/can-periodontal-disease-bea-contributing-factor-for-covid19-severity

24. Gupta S, Mohindra R, Chauhan PK, et al. (2020) SARS-CoV-2 Detection in Gingival Crevicular Fluid. J Dent Res 100(2): pp. 187-193.

25. Kheur S, Kheur M, Gupta AA, et al. (2020) Is the gingival sulcus a potential niche for SARS-Corona virus-2? Med Hypotheses 143(5): pp. 109892

26. Badran Z, Gaudin A, Struillou X, et al. (2020) Periodontal pockets: A potential reservoir for SARS-CoV-2 ? Med Hypotheses 143(5): pp. 42-44.

27. Sampson V, Kamona N, Sampson A (2020) Could there be a link between oral hygiene and the severity of SARS-CoV-2 infections? Br Dent J 228(12): 971-975.

28. Faíco-Filho KS, Passarelli VC, Bellei N (2020) Is higher viral load in SARS-CoV-2 associated with death? Am J Trop Med Hyg 103(5): pp. 2019-2021.

29. Heneghan C, Brassey J, Jefferson T (2020) SARS-CoV-2 viral load and the severity of COVID-19. https://www.cebm.net/ covid-19/sars-cov-2-viral-load-and-the-severity-of-covid-19/

30. Campisi G, Bizzoca ME, Lo Muzio L (2020) A new exciting hypothesis: direct correlation between periodontitis and clinical evolution of COVID-19 patients. https://www.qeios. com/read/WK61AM

31. Schett G, Sticherling M, Neurath MF (2020) COVID-19 : risk for cytokine targeting in chronic inflammatory diseases? Nat Rev Immunol 20(5): pp. 271-272.

32. Sahni V, Gupta S (2020) COVID-19 and Periodontitis: The cytokine connection. Med Hypotheses 144: pp. 109908.

33. Brinkmann V, Reichard U, Goosmann C, et al. (2004) Neutrophil Extracellular Traps Kill Bacteria. Science 303(5663): pp. 1532-1535.

34. Fuchs TA, Abed U, Goosmann C, et al. Novel cell death program leads to neutrophil extracellular traps. J Cell Biol 76(2): pp. 231-241.

35. Sangaletti S, Tripodo C, Chiodoni C, et al. (2012) Neutrophil extracellular traps mediate transfer of cytoplasmic neutrophil antigens to myeloid dendritic cells toward ANCA induction and associated autoimmunity. Blood 120(15): 3007-3018.

36. Demers M, Wagner DD (2014) NETosis: A new factor in tumor progression and cancer-associated thrombosis. Semin Thromb Hemos 40(3): pp. 277-283.

37. Vitkov L, Hartl D, Minnich B, et al. (2017) Janus-faced neutrophil extracellular traps in periodontitis. Front Immunol 8: pp. 1-2.

38. Nakazawa D, Ishizu A (2020) Immunothrombosis in severe COVID-19. EBioMedicine 59: 4-5.

39. Gupta S, Sahni V (2020) The intriguing commonality of NETosis between COVID-19 ampamp; Periodontal disease. Med Hypotheses 144: 109968. 